\title{
Microabrasão dentária para estética em dentes com fluorose dental em criança: relato de caso clínico
}

\author{
Dental microabrasion for esthetics in teeth with dental fluorosis in a child: case report
}

Microabrasión dental para la estética en dientes con fluorosis dental en un niño: reporte de caso

\section{Resumo}

A fluorose acontece quando o flúor é ingerido em pequenas quantidades de maneira crônica, durante a fase de amelogênse dos dentes permanentes, momento em que há menor reabsorção de proteínas, levando à formação de um esmalte dentário mais poroso, caracterizado por estrias esbranquiçadas. Essas alterações vistas simetricamente em dentes homólogos, porque o desenvolvimento intraósseo acontece ao mesmo tempo. Essas estriações podem apresentar nos dentes acometidos de coloração esbranquiçadas até acastanhadas. O objetivo deste trabalho é apresentar uma alternativa para o caso clínico de uma paciente de 10 anos de idade que apresentava fluorose dentária. O planejamento clínico para este caso foi utilizar a técnica da microabrasão no esmalte dentário a base de ácido clorídrico a $6 \%$ e carbeto de sílicio. Apenas uma consulta foi o suficiente para melhorar a estética e alto estima da paciente. Concluímos que a técnica e o material utilizado proporcionaram uma harmonia das manchas de coloração branca e impactou, de forma positiva, a qualidade de vida e alto estima da paciente. Outro ponto a ser considerado é que esta técnica é acessível e de mínima intervenção, uma vez que preservou a estrutura dental não causando nenhum dano ou sensibilidade nos dentes.

Palavras-chave: Microabrasão do esmalte; Fluorose dentária; Estética dentária.

\begin{abstract}
Fluorosis occurs when fluoride is chronically ingested in small amounts, during the amelogenesis phase of permanent teeth, when there is less protein reabsorption, leading to the formation of a more porous tooth enamel, characterized by whitish streaks. These changes are seen symmetrically in homologous teeth, because intraosseous development takes place at the same time. These striations may present whitish to brownish in the affected teeth. The aim of this paper is to present an alternative to the clinical case of a 10-year-old patient who presented with dental fluorosis. The clinical planning for this case was to use the microabrasion technique on tooth enamel based on $6 \%$ hydrochloric acid and silicon carbide. Just one appointment was enough to improve the patient's esthetics and high esteem. We conclude that the technique and the material used provided a harmony of white stains and positively impacted the patient's quality of life and high esteem. Another point to be considered is that this technique is accessible and requires minimal intervention, since it preserved the tooth structure, not causing any damage or sensitivity to the teeth.
\end{abstract}

Keywords: Enamel microabrasion; Dental fluorosis; Esthetics dental. 


\begin{abstract}
Resumen
La fluorosis ocurre cuando el fluoruro se ingiere crónicamente en pequeñas cantidades, durante la fase de amelogénesis de los dientes permanentes, cuando hay menos reabsorción de proteínas, lo que lleva a la formación de un esmalte dental más poroso, caracterizado por vetas blanquecinas. Estos cambios se observan simétricamente en dientes homólogos, porque el desarrollo intraóseo tiene lugar al mismo tiempo. Estas estrías pueden presentarse blanquecinas a parduscas en los dientes afectados. El objetivo de este trabajo es presentar una alternativa al caso clínico de un paciente de 10 años que presentó fluorosis dental. La planificación clínica para este caso fue utilizar la técnica de microabrasión sobre el esmalte dental a base de ácido clorhídrico al $6 \%$ y carburo de silicio. Una sola cita fue suficiente para mejorar la estética y la alta estima del paciente. Concluimos que la técnica y el material utilizado aportaron una armonía de manchas blancas e impactaron positivamente en la calidad de vida y alta estima del paciente. Otro punto a considerar es que esta técnica es accesible y requiere una mínima intervención, ya que preservó la estructura dentaria y no provocó ningún daño ni sensibilidad a los dientes.
\end{abstract}

Palabras clave: Microabrasión del esmalte; Fluorosis dental; Estética dental.

\title{
1. Introdução
}

A fluorose dentária é um distúrbio do desenvolvimento no esmalte, causado por exposições sucessivas a altas concentrações de flúor durante a amelogênse, deixando o esmalte dentário com menor teor de minerais e maior porosidade (Abanto Alvarez et al., 2009; Joaquim et al., 2021). Assim, a faixa etária crítica para o desenvolvimento de fluorose dentária está no período entre 15 a 30 meses na transição da fase secretória, e a fase de maturação do esmalte dos incisivos superiores permanentes (Abanto Alvarez et al., 2009; Cury et al., 2019; Evans \& Darvell, 1995).

Opticamente, a fluorose dentária é caracterizada pela hipomineralização do esmalte dentário, vista como estriações de manchas opacas brancas difusas e simétricas (Fejerskov et al., 1990). Essas manchas, podem ser classificada do grau leve até o grave, sendo que moderada e grave podem afetar adversamente a estética e a qualidade de vida relacionada à saúde bucal (OHRQoL) (Chankanka et al., 2010).

De acordo com o Levantamento das Condições de Saúde Bucal da População Brasileira (SBBrasil) a prevalência média de fluorose dentária é de 9,5\% em crianças de 12 anos e de 5,4 \% em adolescentes de 15 a 19 anos (Saúde, 2011).

Uma ampla gama de intervenções clinica são encontradas na literatura com o propósito para tratar o esmalte fluorótico, que pode variar deste um clareamento externo até a confecção facetas dentárias ou coroas (Akpata, 2001). Como os pacientes com manchas no esmalte são bastante jovens, e hoje, com os avanços nas pesquisas com biomateriais, as abordagens minimamente invasivas, como a microabrasão ganharam ímpeto (Sundfeld et al., 2014).

A microabrasão do esmalte foi inicialmente realizada para a remoção de manchas brancas fluoróticas com ácido clorídrico 36\%, conforme recomendação de Kane em 1926 (Celik et al., 2013), com o tempo e com a preocupação da segurança da técnica, o tempo e a concentração do ácido clorídrico diminui para 18\% (Croll, 1986; Croll \& Cavanaugh, 1986a, 1986b) e atualmente 6\%. (Costa et al., 2021). Esta técnica se limita aas manchas encontradas nas porções mais superficial do esmalte dentário, constituindo um método eficaz, rápido, fácil manejo, com perda de quantidade insignificante do esmalte dentário sadio e não causa prejuízo nos tecidos adjacentes quando realizado de forma correta (Bassir \& Bagheri, 2013; Santana et al., 2018).

O objetivo deste trabalho é relatar um caso clínico da técnica de microabrasão do esmalte como uma alternativa para o tratamento de manchas de fluorose em uma criança de 10 anos de idade que por apresentar fluorose dentária nos dentes permanentes estava a deixando com baixa auto estima.

\section{Relato de Caso}

Este relato foi aprovado pelo Comitê de ética em pesquisa sob o número 03479018.0.0000.5374. 
Paciente B.S.S, sexo feminino, 10 anos de idade, leucoderma, compareceu a clínica de Odontopediatria acompanhada pelo pai que assinou o Termo de consentimento livre e esclarecido. A paciente apresentou histórico odontológico de ortodontia preventiva e queixa de dor e sangramento ao escovar. $\mathrm{Na}$ anamnese, juntamente com o responsável, a paciente relatou que escova os dentes três vezes ao dia, com dentifrício fluoretado em grande quantidade e que fazia ingestão do produto quando criança. Ao realizar o exame clínico foi notado que a paciente apresentava fluorose em todos os dentes, higiene deficiente, apresentando inflamação na gengiva, acúmulo de placa bacteriana em grande quantidade e hipomineralização no elemento 65 (Figura 1a e 1b).

Após o exame clínico, foi realizada a profilaxia com pasta profilática e Pedra Pomes, usando escova de Robson e taça de borracha, em seguida o ICDAS. Foi observado em todos os dentes permanentes alteração de cor esbranquiçado principalmente nas faces incisais/oclusais suspeitando de fluorose dentária. Ao final da consulta inicial, foi realizada orientação de higiene bucal e dieta, juntamente com a entrega de um diário alimentar.

Figura 1a - Exame intra oral inicial. Observa-se a presença de fluorose e higienização bucal inadequada, com gengivas edemaciadas e acúmulo de biofilme dentário.

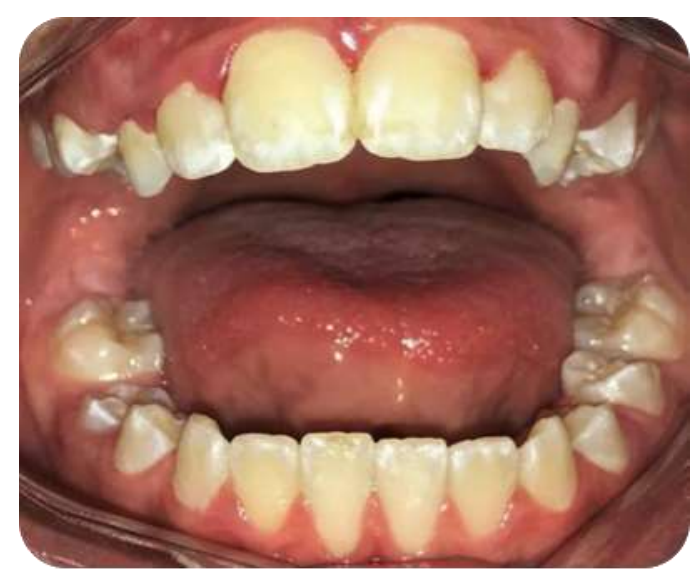

Fonte: Autores.

Figura 1b - Exame intra oral após a profilaxia- nota se gengiva edemaciadas entre os dentes 22 e 23.

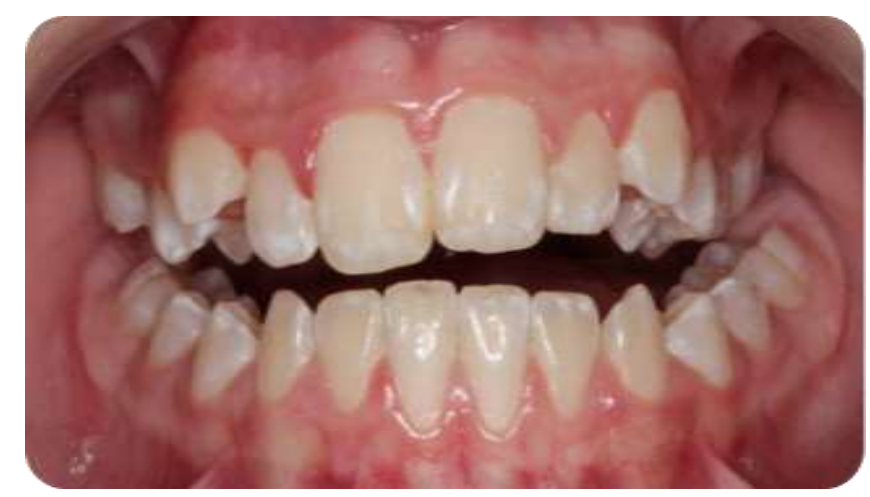

Fonte: Autores.

A profundidade das manchas de fluorose foi realizada pela técnica de transluminação com uso do fotopolimerizado (Figura 2). Quanto mais profunda a mancha, mais opaco ela apresentará e quanto mais superficial menos opaco essa mancha apresentara. 
Figura 2 - Fotopolimerizador para transluminação.

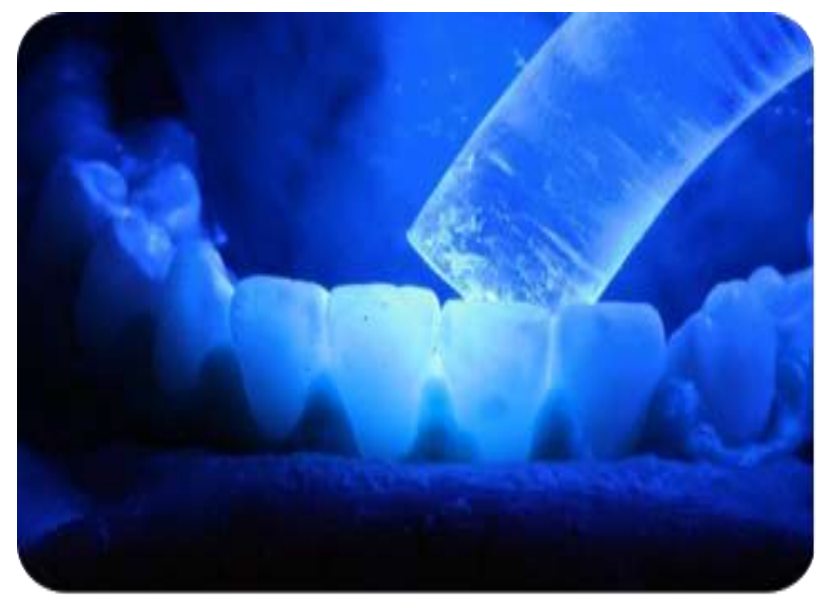

Fonte: Autores.

O tratamento escolhido no presente caso foi de microabrasão realizado com um material clareador (Whitness RM da marca FGM). Para a técnica de microabrasão foi realizada a profilaxia, e, em seguida, isolamento com barreira gengival (Figura 3). E aplicação do produto (Figura 4). Orientações essas de acordo com o fabricante.

Figura 3 - Isolamento com barreira gengival.

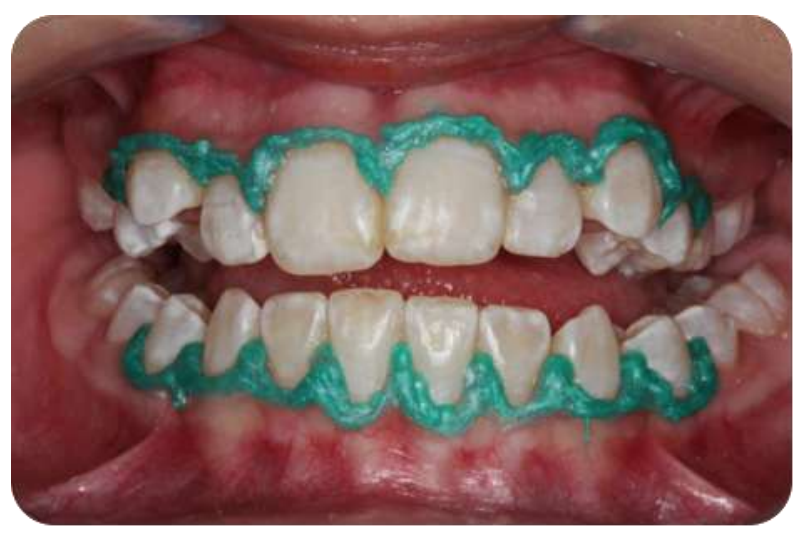

Fonte: Autores.

Figura 4 - Aplicação da pasta microabrasiva.

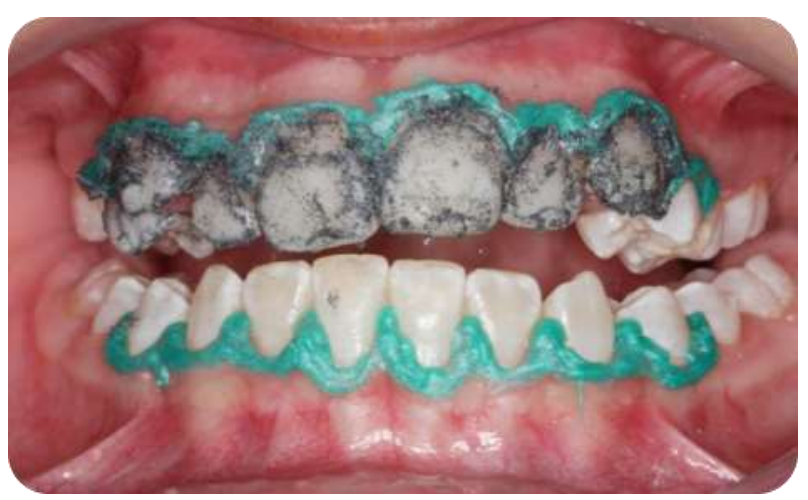

Fonte: Autores. 
Foi realizado em apenas uma sessão com 9 aplicações de 10 segundos em cada dente e logo após lavados abundantemente com a seringa tríplice para a total retirada do produto (figura 5).

Figura 5 - Uso da taça de borracha.

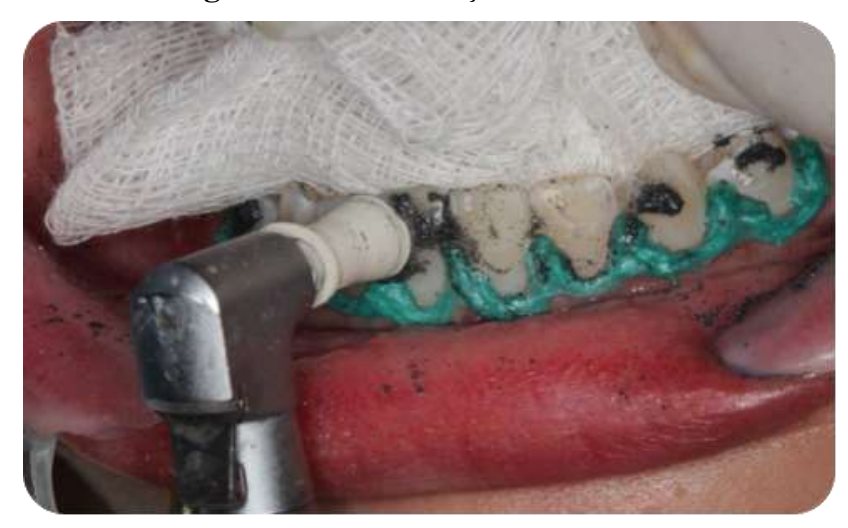

Fonte: Autores.

Figura 6 - Aspecto final do tratamento.

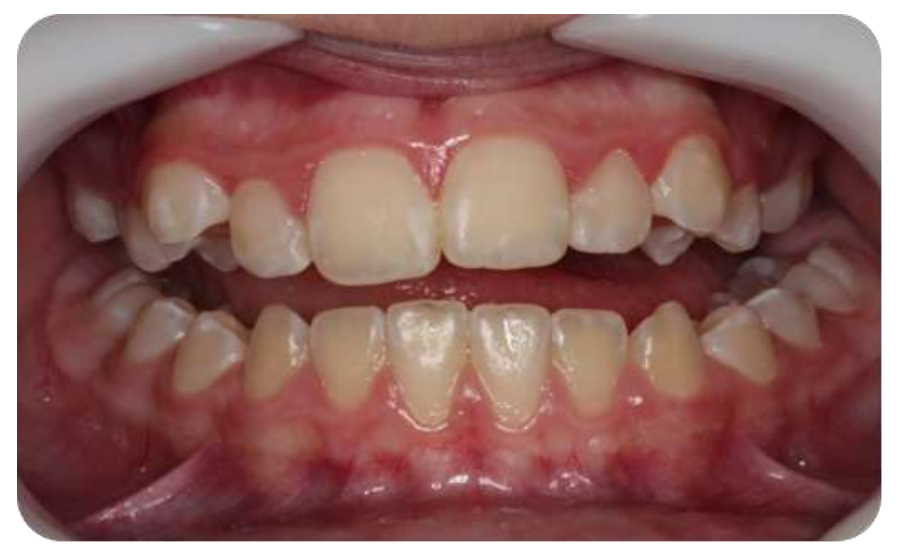

Fonte: Autores.

\section{Discussão}

Mudanças de cores opacas nos dentes podem ocorrer como resultado de danos antes ou depois da erupção dos dentes. Fluorose, hipomineralização devido a trauma e hipominelizações em molares e incisivos podem ser caracterizadas como as lesões opacas de esmalte mais frequentemente observadas na clinica odontológica. Estas condições podem causar problemas estéticos que particularmente afetam crianças e adultos jovens (Chankanka et al., 2010) (Santana et al., 2018).

Sabe se que para o estabelecimento de fluorose dentária é preciso uma ingestão alta e crônica de fluoreto ( $>0,07$ $\mathrm{mg} / \mathrm{F} / \mathrm{kg} / \mathrm{dia}$ ) durante o período de formação do dente, porque o excesso de flúor, afeta metabolicamente os ameloblastos (Abanto Alvarez et al., 2009). Para que ocorra uma condição de alteração severa no esmalte dentário, depende da dose, duração, exposição, estágio da atividade dos ameloblastos e idade do indivíduo. A idade mais crítica está entre os 15 a 30 meses de idade, porque é quando os incisivos superiores permanentes estão na transição entre a fase secretória e a fase de maturação do esmalte e da susceptibilidade individual (Abanto Alvarez et al., 2009; Celeste \& Luz, 2016; Cury et al., 2019; Evans \& Darvell, 1995; Joaquim et al., 2021). No caso clinico aqui descrito está de acordo com a literatura, porque a assim 
como o paciente descrito por Silva et al., 2021, a etiologia da fluorose nessa paciente foi por ter ingerido muita quantidade de pasta de dente fluoretada durante a primeira infância, por achar um sabor agradável para seu paladar.

Quando a fluorose apresenta de forma leve o dente apresentará hipomineralização do esmalte, levando à opacidade, e, podendo apresentar clinicamente na forma de pintas brancas. Uma das técnicas para ajudar no diagnóstico é utilizar a luz do aparelho do fotopolimerizador por palatina ou lingual, para verificar a opacidades das manchas (Lee et al., 2017). Essa técnica é denominada de transluminação e foi realizada nesse caso para determinar o melhor tratamento.

A odontologia atual, preconiza as técnicas e tratamentos conservadores e objetiva a preservação de estrutura dental sadia (Di Giovanni et al., 2018; Shahroom et al., 2019). Um dos fatores de maior desafio para o cirurgião dentista é a resolução estética dos mais variados tipos de manchas presentes no esmalte e a satisfação do paciente, afinal, a estética é uma das principais preocupações dos pacientes adultos, porém as crianças das novas gerações, que nascem conectadas, e se comunicam entre elas pelas redes sociais já apresentam desde cedo preocupação com a estética e sorriso (Silva et al., 2021).

Neste contexto, o aspecto visual causado pela fluorose dental gera desconforto na harmonia estética do sorriso no qual acaba sendo o motivo de consulta odontológica para que se possa realizar tratamentos com o objetivo de disfarçar ou eliminar essas alterações no esmalte (Croll \& Cavanaugh, 1986a).

A paciente desse caso, 10 anos, apresentava manchas superfíciais. E com isso a escolha da técnica da microabrasão conceituado como um procedimento pelo qual uma pequena camada superficial do esmalte, que apresenta alguma forma de alteração - cor, estrutura ou desmineralização -, é removida por meio da ação conjunta de um agente erosivo (ácido) e um agente abrasivo (pedrapomes ou carboneto de silício), expondo-se uma camada mais profunda de esmalte com características normais (Akpata, 2001; Croll \& Cavanaugh, 1986b; Sundfeld et al., 2019).

Assim, de acordo com a literatura e com fabricante do produto, foi realizado em apenas uma sessão com 9 aplicações de 10 segundos em cada dente e logo após lavados abundantemente com a seringa tríplice para a total retirada do produto. Não foi associado o clareamento dentário. Entretanto, estudos afirmam que o nível de satisfação dos pacientes demonstra que a microabrasão do esmalte associada ou não ao clareamento dentário, é considerada um tratamento efetivo para a diminuição das manchas fluoróticas (Chankanka et al., 2010; Costa et al., 2021; Sundfeld et al., 2019). Ao finalizar a primeira consulta, a criança já sentiu feliz com novo aspecto dentário e isso gerou um novo hábito de higiene bucal, porque ela ficou cada vez motivada em cuidar dos dentes. Assim, é muito importante, na clínica odontopediátrica, além de dominar todas as técnicas, ter um correto diagnóstico, sempre motivar o paciente, e seus responsável, para que o tratamento finalize sempre de forma positiva para toda família e para o CD.

\section{Conclusão}

O tratamento de fluorose dentária com a técnica de microabrasão, mostrou-se eficiente no clareando das manchas brancas presentes nos dentes permanentes afetados, preservou estrutura dental proporcionando um resultado estético, satisfatório além de fácil operação e baixo custo, podendo ser uma ótima escolha nesses casos.

\section{Referências}

Abanto Alvarez, J., Rezende, K. M., Marocho, S. M., Alves, F. B., Celiberti, P., \& Ciamponi, A. L. (2009). Dental fluorosis: exposure, prevention and management. Med Oral Patol Oral Cir Bucal, 14(2), E103-107. https://www.ncbi.nlm.nih.gov/pubmed/19179949

Akpata, E. S. (2001, Oct). Occurrence and management of dental fluorosis. Int Dent J, 51(5), 325-333. https://doi.org/10.1002/j.1875-595x.2001.tb00845.x

Bassir, M. M., \& Bagheri, G. (2013). Comparison between phosphoric acid and hydrochloric acid in microabrasion technique for the treatment of dental fluorosis. J Conserv Dent, 16(1), 41-44. https://doi.org/10.4103/0972-0707.105297 
Research, Society and Development, v. 10, n. 14, e07101421759, 2021

(CC BY 4.0) | ISSN 2525-3409 | DOI: http://dx.doi.org/10.33448/rsd-v10i14.21759

Celeste, R. K., \& Luz, P. B. (2016). Independent and Additive Effects of Different Sources of Fluoride and Dental Fluorosis. Pediatr Dent, $38(3), 233-238$. https://www.ncbi.nlm.nih.gov/pubmed/27306248

Celik, E. U., Yildiz, G., \& Yazkan, B. (2013). clinical evaluation of enamel microabrasion for the aesthetic management of mild-to-severe dental fluorosis. $J$ Esthet Restor Dent, 25(6), 422-430. https://doi.org/10.1111/jerd.12052

Chankanka, O., Levy, S. M., Warren, J. J., \& Chalmers, J. M. (2010). A literature review of aesthetic perceptions of dental fluorosis and relationships with psychosocial aspects/oral health-related quality of life. Community Dent Oral Epidemiol, 38(2), 97-109. https://doi.org/10.1111/j.1600-0528.2009.00507.x

Costa, D., Meireles, A., Ferreira, J. L., Alcantara, P., Torres, L. A., Camara, J. V. F., Pierote, J. J., Canuto, M. H., \& Araujo, C. T. (2021). Correction to: Microabrasion effect on enamel susceptibility to penetration of hydrogen peroxide: an experimental and computational study. Odontology, 109(4), 779. https://doi.org/10.1007/s10266-021-00606-3

Croll, T. P. (1986. Enamel color improvement: all things considered. Quintessence Int, 17(5), 271-275. https://www.ncbi.nlm.nih.gov/pubmed/3460108

Croll, T. P., \& Cavanaugh, R. R. (1986a). Enamel color modification by controlled hydrochloric acid-pumice abrasion. II. Further examples. Quintessence Int, 17(3), 157-164. https://www.ncbi.nlm.nih.gov/pubmed/3458266

Croll, T. P., \& Cavanaugh, R. R. (1986b). Hydrochloric acid-pumice enamel surface abrasion for color modification: results after six months. Quintessence Int, 17(6), 335-341. https://www.ncbi.nlm.nih.gov/pubmed/3014596

Cury, J. A., Ricomini-Filho, A. P., Berti, F. L. P., \& Tabchoury, C. P. (2019). Systemic Effects (Risks) of Water Fluoridation. Braz Dent J, 30(5), 421-428 https://doi.org/10.1590/0103-6440201903124

Di Giovanni, T., Eliades, T., \& Papageorgiou, S. N. (2018). Interventions for dental fluorosis: A systematic review. J Esthet Restor Dent, 30(6), 502-508. https://doi.org/10.1111/jerd.12408

Evans, R. W., \& Darvell, B. W. (1995). Refining the estimate of the critical period for susceptibility to enamel fluorosis in human maxillary central incisors. $J$ Public Health Dent, 55(4), 238-249. https://doi.org/10.1111/j.1752-7325.1995.tb02376.x

Fejerskov, O., Manji, F., \& Baelum, V. (1990). The nature and mechanisms of dental fluorosis in man. J Dent Res, 69 Spec No, 692-700; discussion 721. https://doi.org/10.1177/00220345900690S135

Joaquim, B. F., Mansano T., Parreiras, S. O., \& Sônego, M. V. (2021). Esthetic resolution of enamel white spot lesion trough the association of conservative techniques to resin infiltration: Case report. Research, Society and Development, 10(8), e6010817063. https://doi.org/10.33448/rsd-v10i8.17063

Lee, R. C., Jang, A., \& Fried, D. (2017). Near-infrared imaging of enamel hypomineralization due to developmental defects. Proc SPIE Int Soc Opt Eng, 10044. https://doi.org/10.1117/12.2256768

Santana, R. M., Santos, L. T. B. d., Imparato, J. C. P., \& Rezende, K. M. (2018). Microabrasão de esmalte- caso clínico para estética na primeira infância. Revista da APCD, 72(1), 4

Saúde, B. M. d. (2011). SB Brasil 2010: Pesquisa Nacional de Saúde bucal: resultados principais. Ministério da Saúde.

Shahroom, N. S. B., Mani, G., \& Ramakrishnan, M. (2019, Oct). Interventions in management of dental fluorosis, an endemic disease: A systematic review. $J$ Family Med Prim Care, 8(10), 3108-3113. https://doi.org/10.4103/jfmpc.jfmpc 648

Silva, M. R. M. d. S., Neto, A. C. C. L. d. C., Santos, L. K. G. d., \& Silva, A. M. F. d. (2021). Tratamento de fluorose dentária causada por dentifrício com a técnica de microabrasão de esmalte: relato de caso clínico. Research, Society and Development, 10(7), 6.

Silva, M. V. d., Bussadori, S. K., Santos, E. M., \& Rezende, K. M. (2021). Behaviour management of the contemporary child in paediatric dentistry: an overview of the research. Pesquisa Brasileira Odontoped Clin Integr, 21, 12. https://doi.org/https://doi.org/10.1590/pboci.2021.090

Sundfeld, D., Pavani, C. C., Pini, N., Machado, L. S., Schott, T. C., \& Sundfeld, R. H. (2019). Enamel Microabrasion and Dental Bleaching on Teeth Presenting Severe-pitted Enamel Fluorosis: A Case Report. Oper Dent, 44(6), 566-573. https://doi.org/10.2341/18-116-T

Sundfeld, R. H., Sundfeld-Neto, D., Machado, L. S., Franco, L. M., Fagundes, T. C., \& Briso, A. L. (2014). Microabrasion in tooth enamel discoloration defects: three cases with long-term follow-ups. $J$ Appl Oral Sci, 22(4), 347-354. https://doi.org/10.1590/1678-775720130672 